\title{
Self-Healing Solid Polymer Electrolyte for Room- Temperature Solid-State Lithium Metal Batteries
}

Lanshuang Zhang, ${ }^{a, b, \#}$ Panpan Zhang, ${ }^{a, b, \#}$ Caiyun Chang, ${ }^{a, c}$ Wenbin Guo, ${ }^{a, b}$ Zi Hao Guo, ${ }^{a, b}$ Xiong $P u^{a, b, c, d, *}$

${ }^{\text {a }}$ CAS Center for Excellence in Nanoscience, Beijing Key Laboratory of Micro-Nano Energy and Sensor, Beijing Institute of Nanoenergy and Nanosystems, Chinese Academy of Sciences, Beijing 101400, China

${ }^{\mathrm{b}}$ School of Nanoscience and Technology, University of Chinese Academy of Sciences.

Beijing 100049, China

${ }^{\mathrm{c}}$ Center on Nanoenergy Research, School of Chemistry and Chemical Engineering, School of Physical Science and Technology, Guangxi University, Nanning 530004, China

${ }^{\mathrm{d}}$ CUSTech Institute of Technology, Wenzhou, Zhejiang, 325024, China

\# These authors contributed equally.

*Corresponding author. E-mail: puxiong@binn.cas.cn 


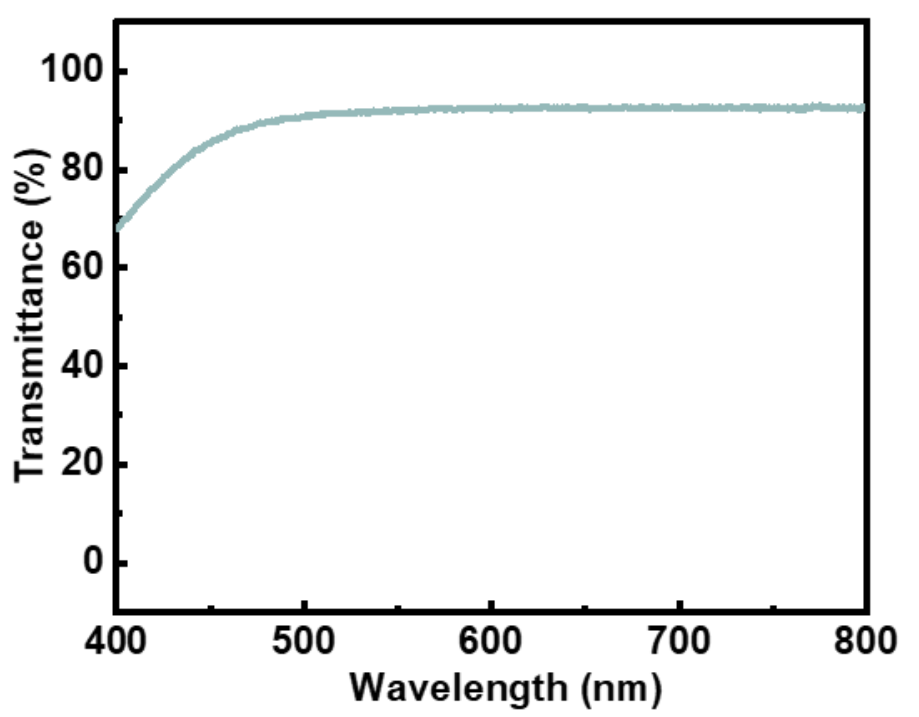

Figure S1. Transmittance in the visible range for SHSPE (at $8.5 \mathrm{~mm}$ thickness).

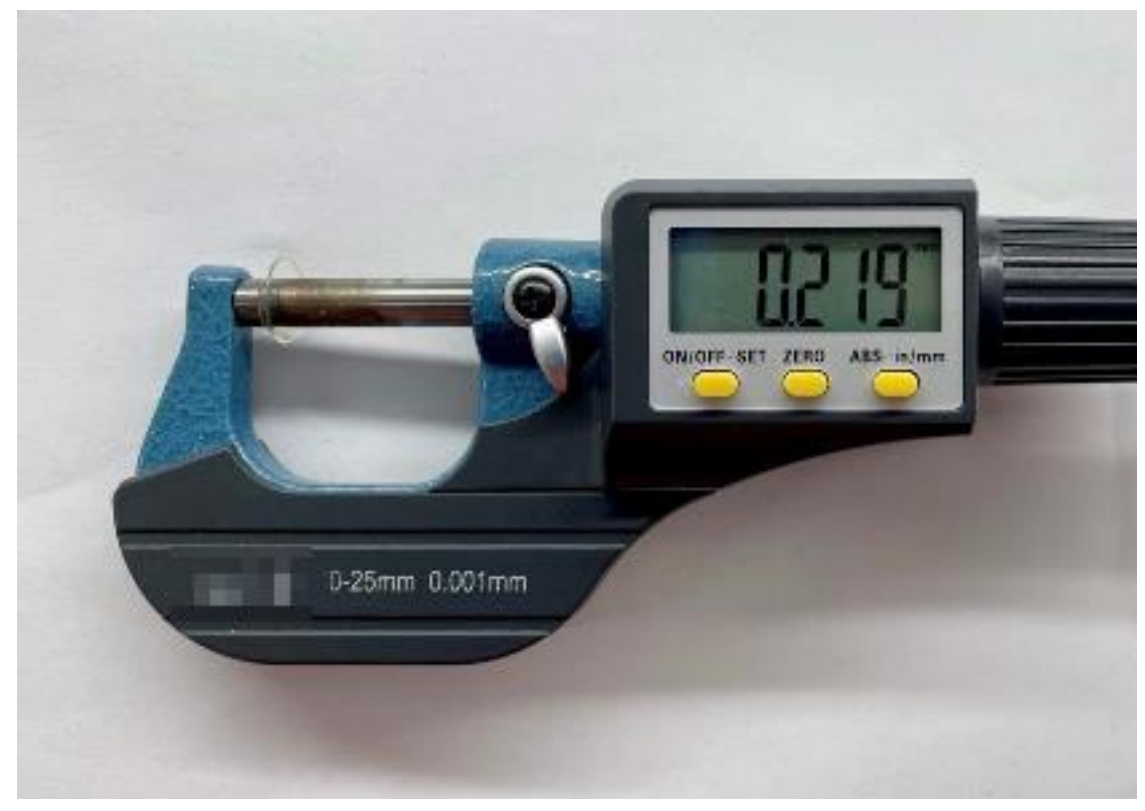

Figure S2. Digital photo of the thickness of SHSPE. 


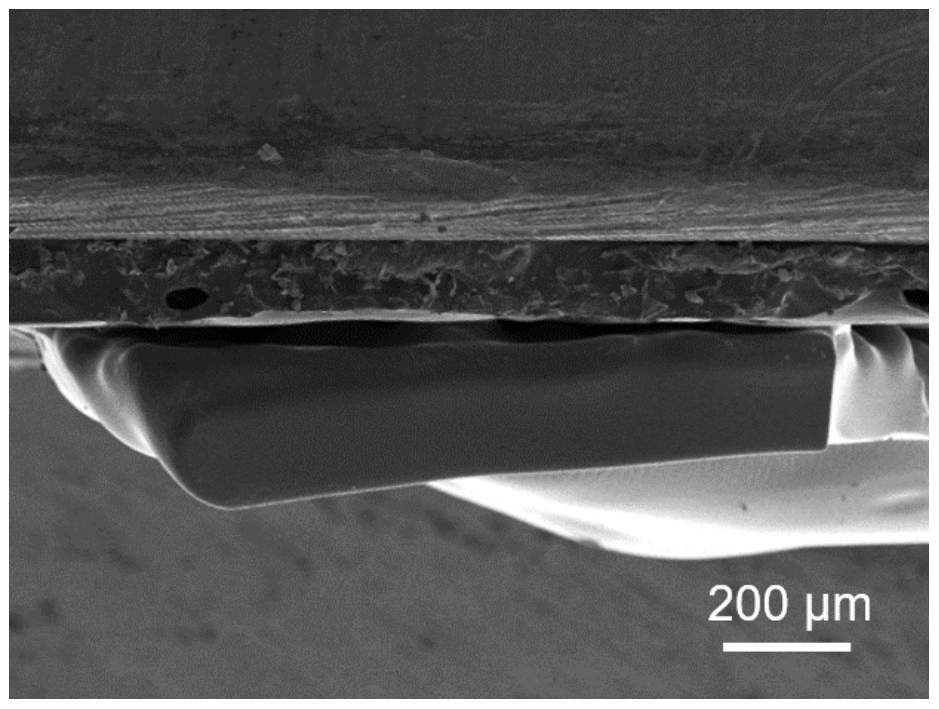

Figure S3. 90 degree cross-sectional SEM images.
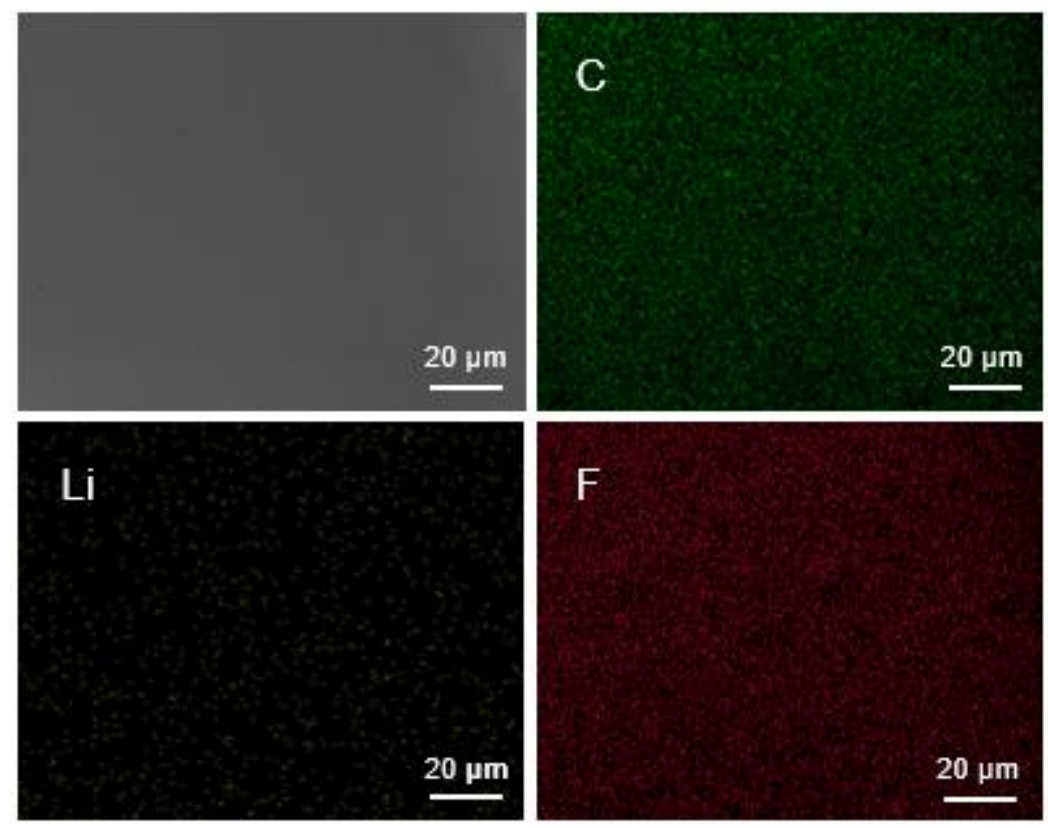

Figure S4. SEM image and EDS-mapping analysis of C, Li, and F elements for SHSPE. 


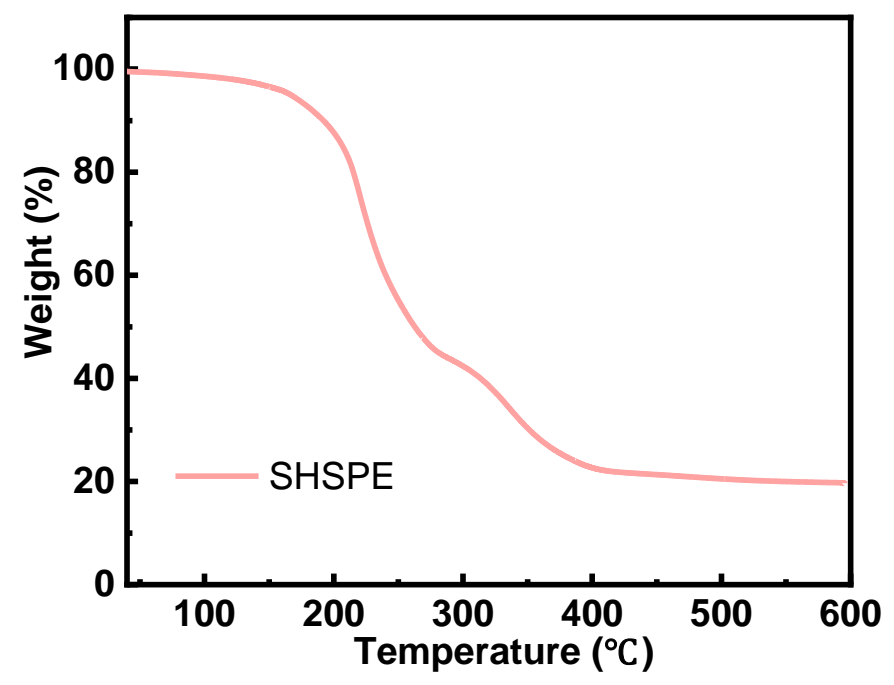

Figure S5. TGA of the SHSPE.

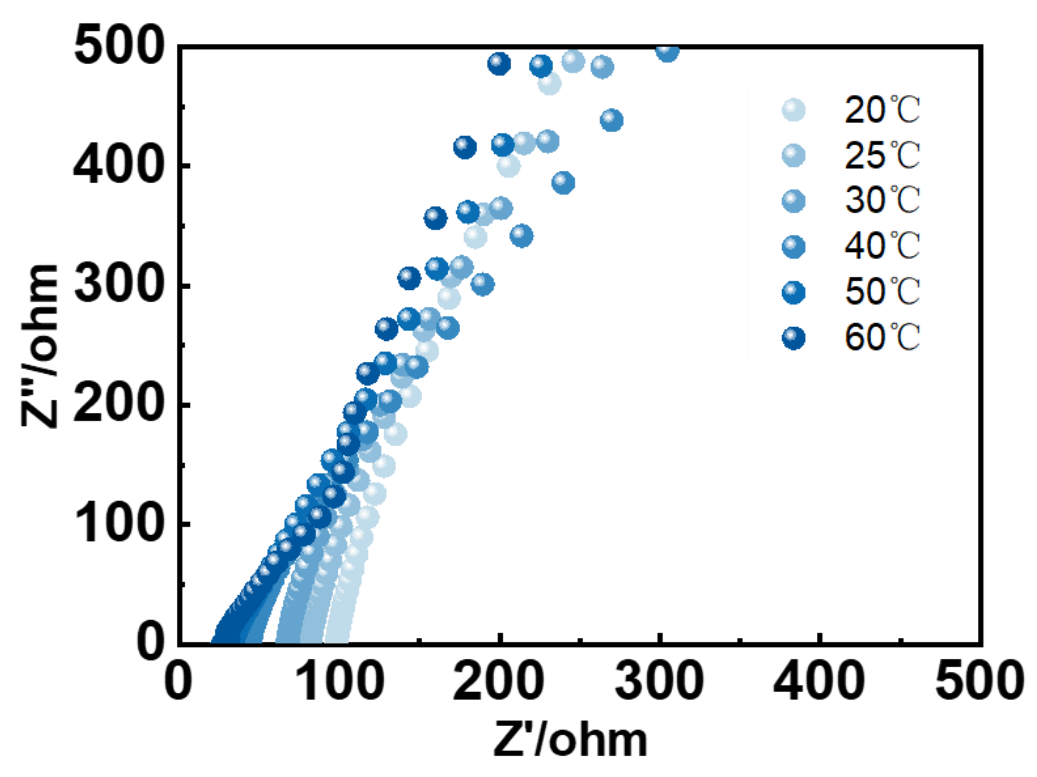

Figure S6. The EIS plots of stainless steel|original SHSPE|stainless steel with various temperatures 


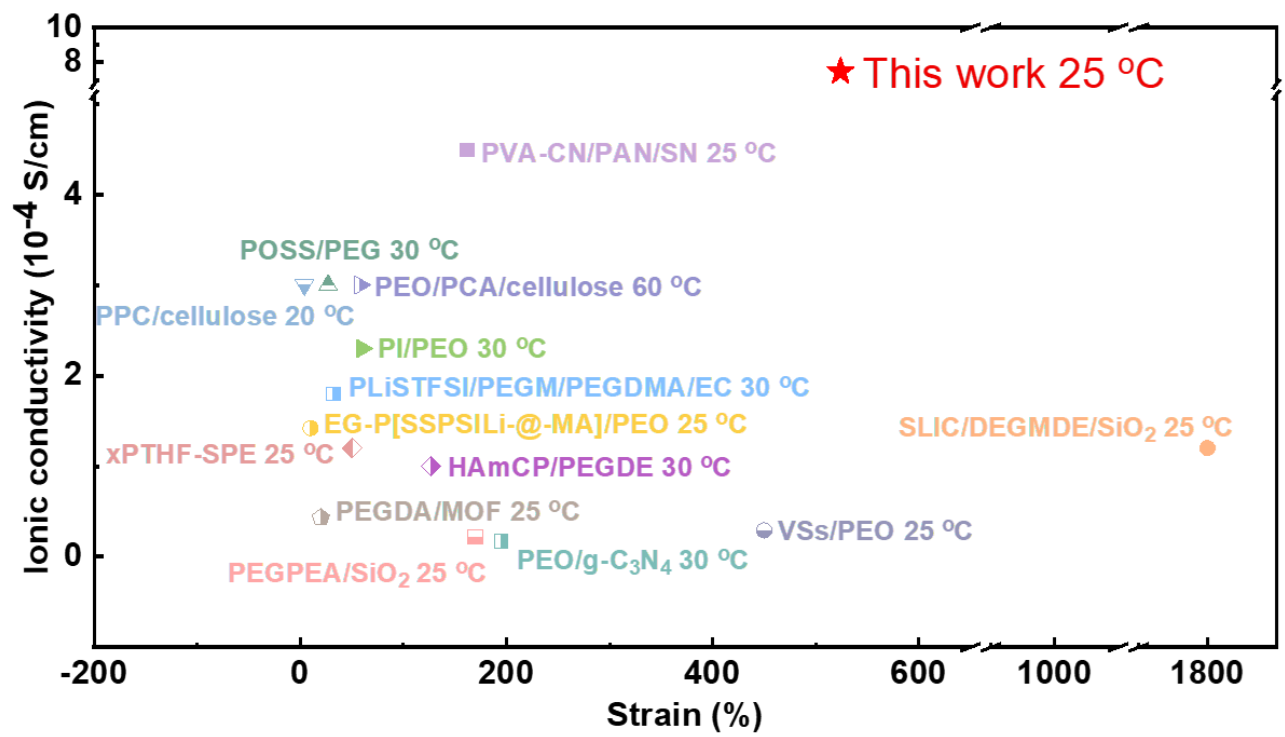

Figure S7. Comparison of the ionic conductivity and stretchability of the SHSPE to other other polymer electrolytes reported in the literature. ${ }^{1-14}$

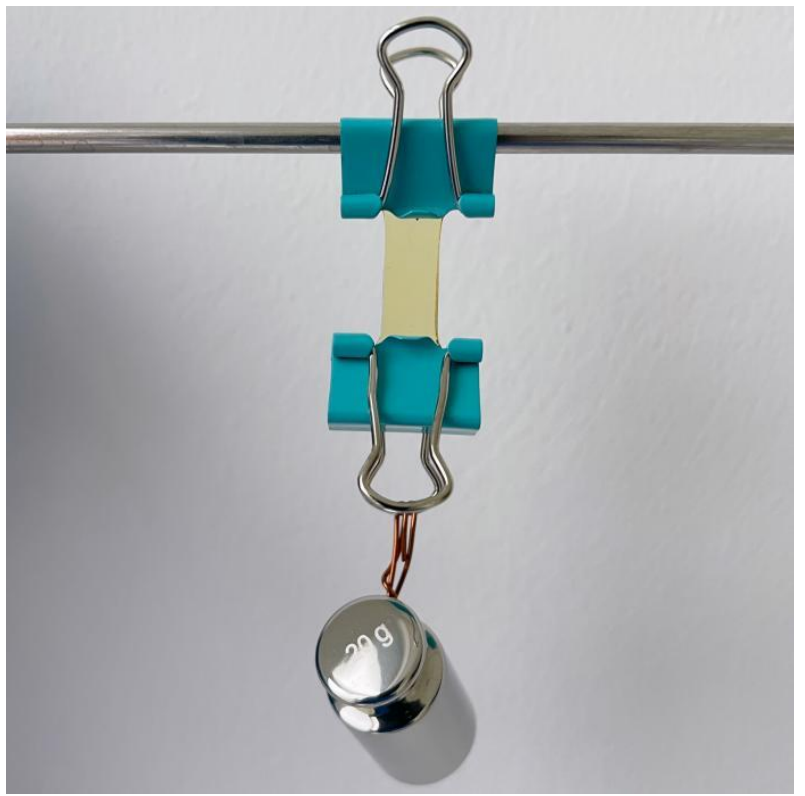

Figure S8. Photograph showing that SHSPE under the hanging weight of $20 \mathrm{~g}$. 


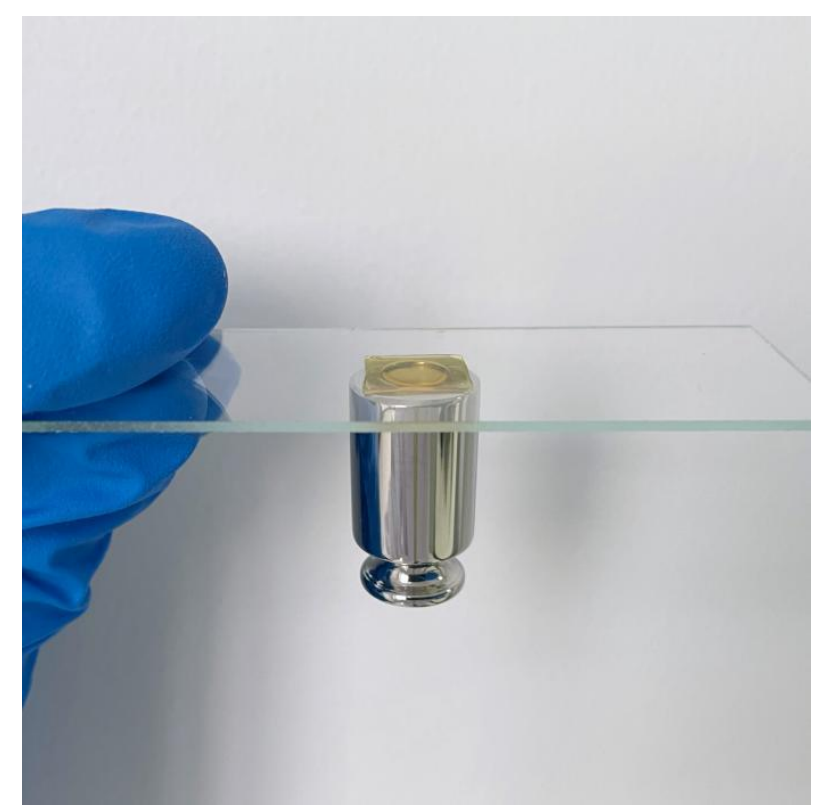

Figure S9. Photo image of the SHSPE attached to a glass surface to completely support $20 \mathrm{~g}$ of counterweight.

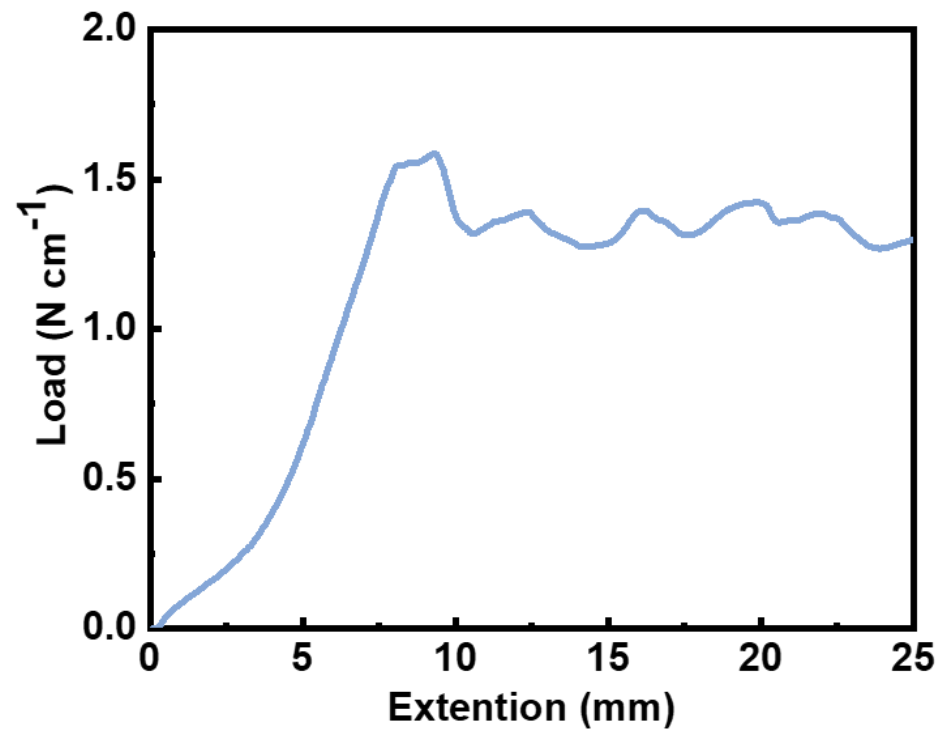

Figure S10. Peeling test of the SHSPE with lithium metal. 


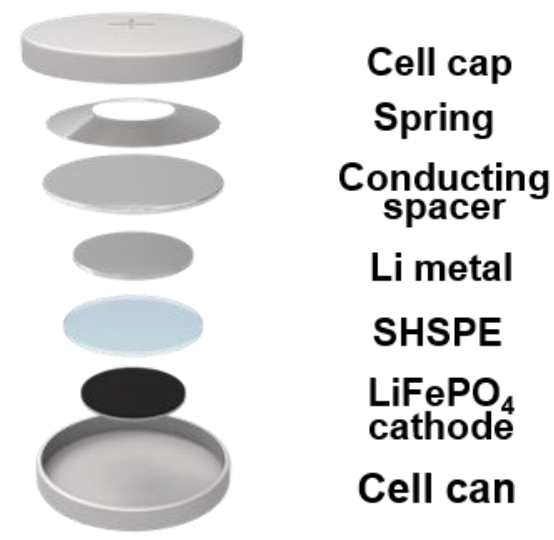

Figure S11. Illustration of the coin cell featuring Li metal, SHSPE membrane, and a $\mathrm{LiFePO}_{4}$ cathode vertically stacked in it.

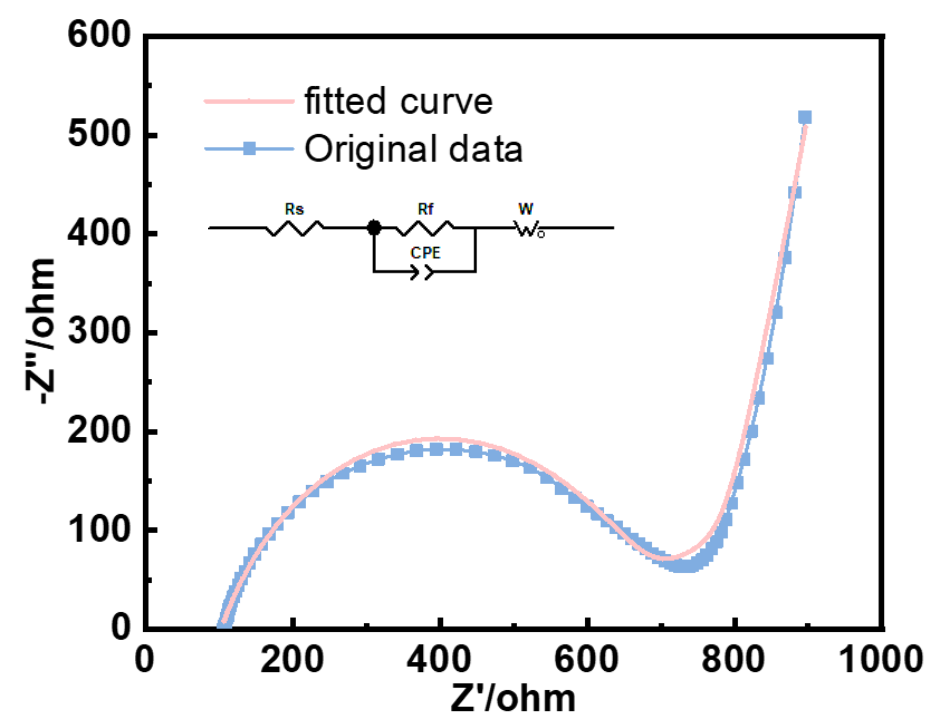

Figure S12. EIS spectra and the equivalent fitting circuit (inset) of $\mathrm{Li}|\mathrm{SHSPE}| \mathrm{LiFePO}$ cell at 0th. 


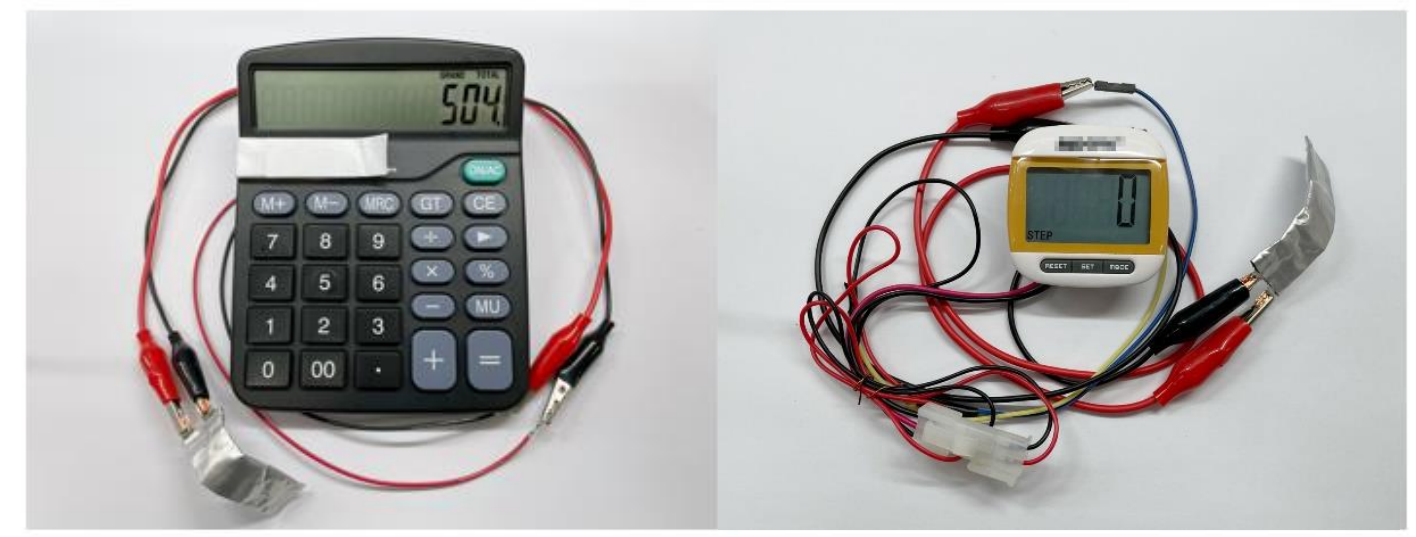

Figure S13. Photographs images of the $\mathrm{Li}|\mathrm{SHSPE}| \mathrm{LiFePO} 4$ pouch cell power a calculator and a pedometer.

\section{REFERENCES}

(1) Cao, C.; Li, Y.; Chen, S.; Peng, C.; Li, Z.; Tang, L.; Feng, Y.; Feng, W. ElectrolyteSolvent-Modified Alternating Copolymer as a Single-Ion Solid Polymer Electrolyte for High-Performance Lithium Metal Batteries. ACS Appl. Mater. Interfaces 2019, 11, $35683-35692$.

(2) Wang, Z.; Wang, S.; Wang, A.; Liu, X.; Chen, J.; Zeng, Q.; Zhang, L.; Liu, W.; Zhang, L. Covalently Linked Metal-Organic Framework (MOF)-Polymer All-SolidState Electrolyte Membranes for Room Temperature High Performance Lithium Batteries. J. Mater. Chem. A 2018, 6, 17227-17234.

(3) Mackanic, D. G.; Yan, X.; Zhang, Q.; Matsuhisa, N.; Yu, Z.; Jiang, Y.; Manika, T.; Lopez, J.; Yan, H.; Liu, K.; Chen, X.; Cui, Y.; Bao, Z. Decoupling of Mechanical Properties and Ionic Conductivity in Supramolecular Lithium Ion Conductors. Nat. Commun. 2019, 10, 5384.

(4) Huang, Z.; Pan, Q.; Smith, D. M.; Li, C. Y. Plasticized Hybrid Network Solid Polymer Electrolytes for Lithium-Metal Batteries. Adv. Mater. Interfaces 2019, 6, 1-8.

(5) Wan, J. Y.; Xie, J.; Kong, X.; Liu, Z.; Liu, K.; Shi, F. F.; Pei, A.; Chen, H.; Chen, W.; Chen, J.; Zhang, X. K.; Zong, L. Q.; Wang, J. Y.; Chen, L. Q.; Qin, J.; Cui, Y. 
Ultrathin, Flexible, Solid Polymer Composite Electrolyte Enabled with Aligned Nanoporous Host for Lithium Batteries. Nat. Nanotechnol. 2019, 14, 705

(6) Zhang, J. J.; Yue, L. P.; Hu, P.; Liu, Z. H.; Qin, B. S.; Zhang, B.; Wang, Q. F.; Ding, G. L.; Zhang, C. J.; Zhou, X. H.; Yao, J. H.; Cui, G. L.; Chen, L. Q. Taichi-Inspired Rigid-Flexible Coupling Cellulose-Supported Solid Polymer Electrolyte for HighPerformance Lithium Batteries. Sci. Rep. 2014, 4, 7.

(7) Sun, Z.; Li, Y.; Zhang, S.; Shi, L.; Wu, H.; Bu, H.; Ding, S. G-C3N4 Nanosheets Enhanced Solid Polymer Electrolytes with Excellent Electrochemical Performance, Mechanical Properties, and Thermal Stability. J. Mater. Chem. A 2019, 7, 1106911076.

(8) Zuo, C.; Yang, M.; Wang, Z.; Jiang, K.; Li, S.; Luo, W.; He, D.; Liu, C.; Xie, X.; Xue, Z. Cyclophosphazene-Based Hybrid Polymer Electrolytes Obtained via Epoxy Amine Reaction for High-Performance All-Solid-State Lithium-Ion Batteries. J. Mater. Chem. A 2019, 7, 18871-18879.

(9) Tang, W. J.; Tang, S.; Zhang, C. J.; Ma, Q. T.; Xiang, Q.; Yang, Y. W.; Luo, J. Y. Simultaneously Enhancing the Thermal Stability, Mechanical Modulus, and Electrochemical Performance of Solid Polymer Electrolytes by Incorporating 2D Sheets. Adv. Energy Mater. 2018, 8, 7.

(10) Niu, C.; Liu, J.; Chen, G.; Liu, C.; Qian, T.; Zhang, J.; Cao, B.; Shang, W.; Chen, Y.; Han, J.; Du, J.; Chen, Y. Anion-Regulated Solid Polymer Electrolyte Enhances the Stable Deposition of Lithium Ion for Lithium Metal Batteries. J. Power Sources 2019, 417, 70-75.

(11) Mackanic, D. G.; Michaels, W.; Lee, M.; Feng, D.; Lopez, J.; Qin, J.; Cui, Y.; Bao, Z. Crosslinked Poly(Tetrahydrofuran) as a Loosely Coordinating Polymer Electrolyte. Adv. Energy Mater. 2018, 8, 1800703.

(12) Zhou, D.; He, Y.-B.; Liu, R.; Liu, M.; Du, H.; Li, B.; Cai, Q.; Yang, Q.-H.; Kang, F. In Situ Synthesis of a Hierarchical All-Solid-State Electrolyte Based on Nitrile Materials for High-Performance Lithium-Ion Batteries. Adv. Energy Mater. 2015, 5, 1500353.

(13) Zhang, J.; Zhao, J.; Yue, L.; Wang, Q.; Chai, J.; Liu, Z.; Zhou, X.; Li, H.; Guo, Y.; Cui, G.; Chen, L. Safety-Reinforced Poly(Propylene Carbonate)-Based All-Solid-State Polymer Electrolyte for Ambient-Temperature Solid Polymer Lithium Batteries. $A d v$. Energy Mater. 2015, 5, 1501082. 
(14) Luo, G.; Yuan, B.; Guan, T.; Cheng, F.; Zhang, W.; Chen, J. Synthesis of Single Lithium-Ion Conducting Polymer Electrolyte Membrane for Solid-State Lithium Metal Batteries. ACS Appl. Energy Mater. 2019, 2, 3028-3034. 